\title{
Relativistic Unmagnetized Collisionless Shocks Propagating into the Inhomogeneous Media
}

\author{
S. Tomita* and Y. Ohira \\ Department of Physics and Mathematics, Aoyama Gakuin University, Japan \\ E-mail: tomisaraephys.aovama.ac.jp, ohiraephys.aovama.ac.ip
}

The Weibel instability generates magnetic fields in a collisionless plasma with anisotropic temperature, which is thought to be a crucial role for particle acceleration and magnetic field generation in relativistic collisionless shocks. According to observations of afterglows of Gamma-ray Busts (GRBs), magnetic fields are strongly amplified to about 100 times the shock-compressed value in the large downstream region of the relativistic shock. However, recent simulations of collisionless shocks in homogeneous plasmas suggest that the magnetic field generated by the Weibel instability decays rapidly, which cannot explain observed properties of afterglows of GRBs. In reality, there must be density fluctuations. We proposed a new model for the magnetic field generation in the far downstream region of the relativistic shock. Relativistic shocks propagating to inhomogeneous plasmas make an anisotropic density structure in the downstream region. Then, an anisotropic velocity distribution is generated, so that the magnetic field is generated by the Weibel instability in the far downstream region (S. Tomita \& Y. Ohira, ApJ, 2016). In this study, we perform Particle-In-Cell (PIC) simulations of relativistic unmagnetized collisionless shocks propagating into the inhomogeneous plasma to investigate whether or not our model actually work. The simulation shows that there is a larger temperature anisotropy in the far downstream region compared with a uniform case. The observed temperature anisotropy is sufficiently large to generate the required magnetic field. In oder to see the Weibel instability by the observed anisotropy, we need larger simulations.

35th International Cosmic Ray Conference - ICRC2017

10-20 July, 2017

Bexco, Busan, Korea

${ }^{*}$ Speaker. 


\section{Introduction}

Colissionless shocks formed in the high-energy astrophysical phenomena are thought to be sites for particle acceleration and generation of electromagnetic fields. In particular for relativistic shocks, the Weibel instability is excited by plasmas with anisotropic velocity distribution [प]]] and generates magnetic field fluctuations, although it also occurs in nonrelativistic shocks [四, 目]. Therefore, the Weibel instability is thought to be important for the particle acceleration and generation of magnetic fields [ [ $\mathbb{W}$ ]. Observations of afterglows of Gamma-ray bursts (GRBs) show that the magnetic field is amplified to 100 times the shock-compressed values in a large downstream region [10, [3]], although the amplification of magnetic fields is not needed for some GRB afterglows [5]. The Weibel instability has been investigated by Particle-In-Cell (PIC) simulations to understand its non-linear evolution. Recent simulations of collisionless shocks in homogeneous plasmas show that the magnetic field generated by the Weibel instability decays rapidly, which cannot explain observed properties of afterglows of GRBs. There really must be density fluctuations in the interstellar medium (ISM). We proposed a new model for the magnetic field generation in the far downstream region of the relativistic collisionless shock. Relativistic shocks propagating to an inhomogeneous plasma make an anisotropic density structure in the downstream region. Then, particles anisotropically escape from the high density region, so that an anisotropic velocity distribution is generated. As a result, the magnetic field is generated by the Weibel instability in the far downstream region [9]. In this works, by means of two-dimensional PIC simulation, we investigate whether our model works in the far downstream region of the relativistic collisionless shock propagating to the inhomogeneous media.

\section{Simulation Setup}

We use the two-dimensional electromagnetic PIC code, pCANS, [8] to simulate an unmagnetized relativistic shock propagating to an inhomogeneous electron-positron plasma. To suppress the numerical Cherenkov instability (NCI)[B] , we modified the original code of pCANS. In the modified code, Maxwell's equations are solved by the explicit method with the forth order accuracy in space [四]. Furthermore, we used the four-pass filtering for the current and electromagnetic fields [2]. We set a two-dimensional simulation box in the $x-y$ plane with periodic boundary condition in the y-direction. We use electron-positron plasmas in this simulation. The simulation box size is $L_{\mathrm{x}}=4 \times 10^{3} c / \omega_{\mathrm{p}}, L_{\mathrm{y}}=8 \times 10^{2} c / \omega_{\mathrm{p}}$, where $\omega_{\mathrm{p}}$ and $\mathrm{c}$ are the plasma frequency defined by the number density of the upstream electron-positron plasma in the downstream rest frame and the speed of light, respectively. The cell size and time step are $\Delta \mathrm{x}=\Delta \mathrm{y}=0.1 c / \omega_{\mathrm{p}}$ and $\Delta t=0.05 \omega_{\mathrm{p}}^{-1}$, respectively. The upstream electron-positron plasma has a Lorentz factor of $\gamma=2$ and thermal velocity of $v_{\text {th }}=0.1 c$. They are injected at the left boundary $(x=0)$ and reflected at the right boundary $\left(x=4 \times 10^{3} c / \omega_{\mathrm{p}}\right)$. In this boundary condition, a collisionless shock propagates to the upstream region with a shock velocity of $\overrightarrow{v_{\mathrm{sh}}} \approx-c / 3 \vec{x}$, that is, the simulation is performed in the downstream rest frame. The initial spatial distribution of the electron-positron plasma in the upstream region is given by

$$
n(x, y)=n_{0}\left[1+0.5 \sin \left(2 \pi x / \lambda_{\mathrm{x}}\right) \sin \left(2 \pi y / \lambda_{\mathrm{y}}\right)\right],
$$

where $n_{0}$ is the mean number density and we set $\lambda_{\mathrm{x}}=4 \times 10^{2} c / \omega_{p}, \lambda_{\mathrm{y}}=2 \lambda_{\mathrm{x}}$ in this simulation. 


\section{Simulation Result}

The following figures are snapshots of the simulation at the $\omega_{\mathrm{p}} t=4 \times 10^{3}$. In Fig. 四 and 凹, we show that the spatial distribution of the plasma density normalized by the mean upstream plasma density and the average one over the y-direction, respectively. The mean downstream density is compressed to about three times the mean upstream density in the shock transition region (Fig. (2)). In order to confirm that whether our model works in this simulation, we investigate the spatial evolution of a temperature anisotropy $T_{\mathrm{y}} / T_{\mathrm{x}}-1$ (Fig. B]). For the inhomogeneous case, the downstream region keeps the anisotropy of about 0.05 while it is almost zero for the homogeneous case. We show the spatial distribution of the magnetic field energy density normalized by the mean upstream kinetic energy density, $\varepsilon_{\mathrm{B}}$, in Fig. 田. The magnetic fields are strongly amplified in the shock transition region. However, as shown in Fig. [1, $\varepsilon_{\mathrm{B}}$ averaged over the y-direction simply decays in the downstream region unlike the expectation of our model. Fig. $\amalg(b)$ and $\Psi(b)$ are the enlarged views of the region at $2400 c / \omega_{\mathrm{p}} \leq x \leq 3000 c / \omega_{\mathrm{p}}$, respectively. These show the filamentary structures in the shock upstream region.
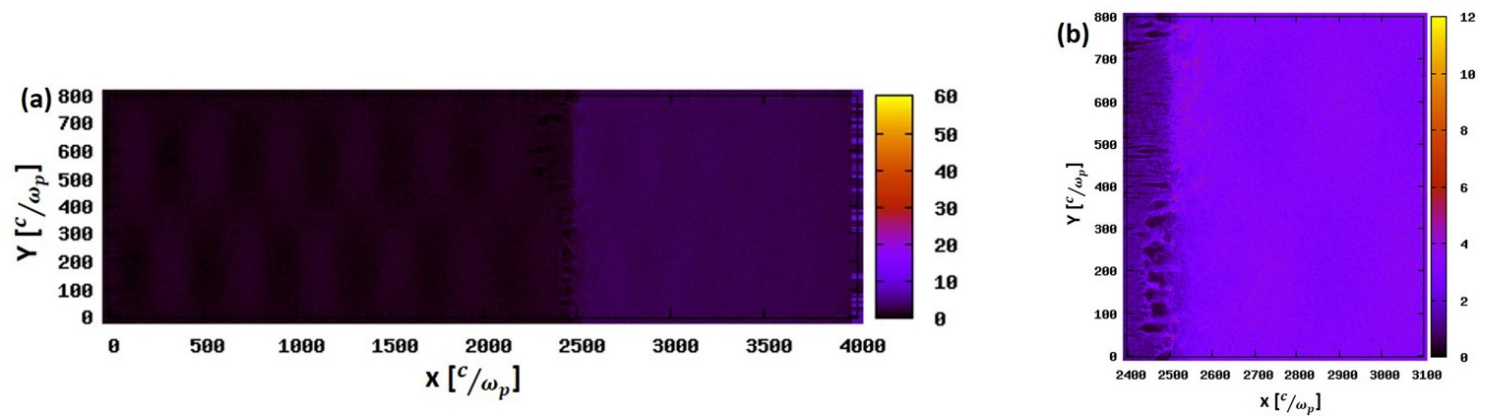

Figure 1: Panel (a): Spatial distribution of the plasma density normalized by the mean upstream plasma density at $\omega_{\mathrm{p}} t=4000$. Panel (b): The enlarged view of the region at $2400 \mathrm{c} / \omega_{\mathrm{p}} \leq x \leq 3100 \mathrm{c} / \omega_{\mathrm{p}}$. 


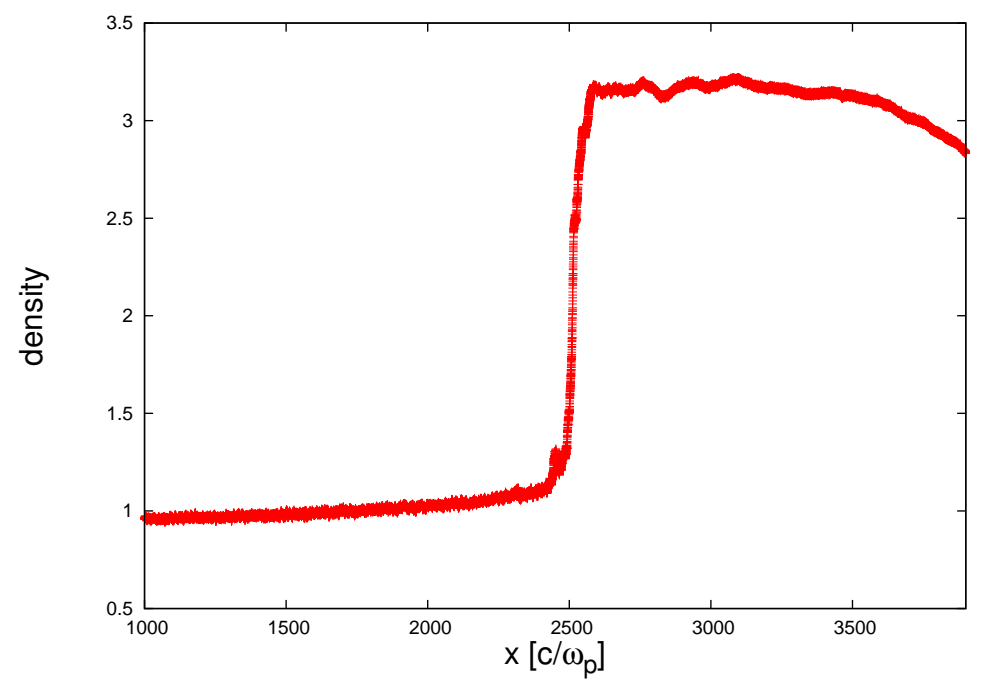

Figure 2: Spatial profile of the plasma density averaged over the y-direction at $\omega_{\mathrm{p}} t=4000$. The density is normalized by the mean upstream plasma density.

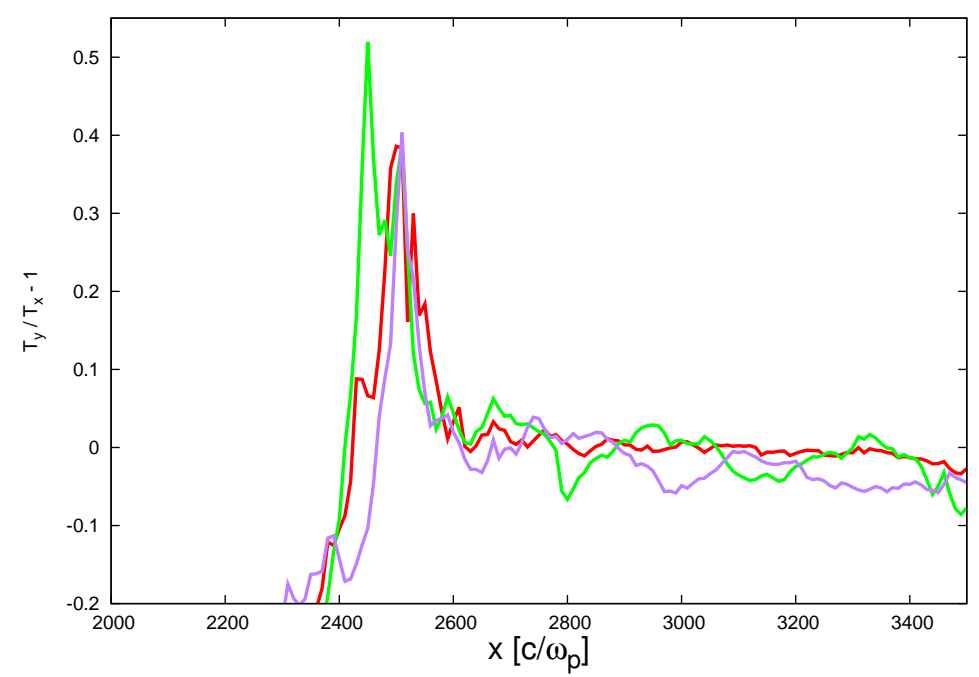

Figure 3: Spatial profile of the temperature anisotropy $T_{\mathrm{y}} / T_{\mathrm{x}}-1$ at $\omega_{\mathrm{p}} t=4000$. The red curve is the homogeneous cases. The green and purple curves represent the anisotropy profile at the different position in the $y$-direction of the inhomogeneous case, respectively. 

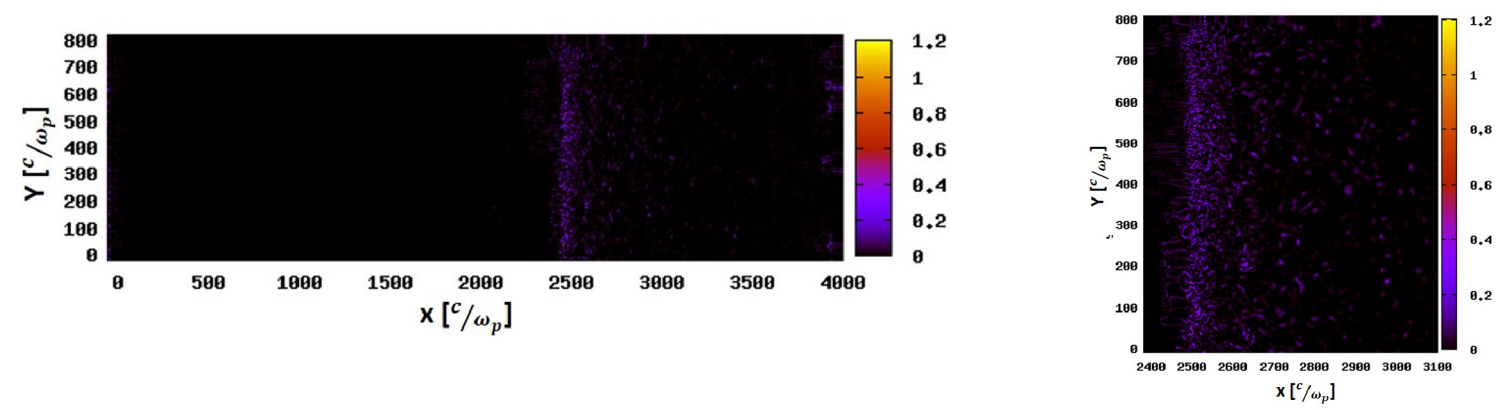

Figure 4: Panel (a): Spatial distribution of the magnetic field energy density normalized by the mean upstream kinetic energy density, $\varepsilon_{\mathrm{B}}$ at $\omega_{\mathrm{p}} t=4000$. Panel (b): The enlarged view of the region at $2400 c / \omega_{\mathrm{p}} \leq x \leq 3100 c / \omega_{\mathrm{p}}$.

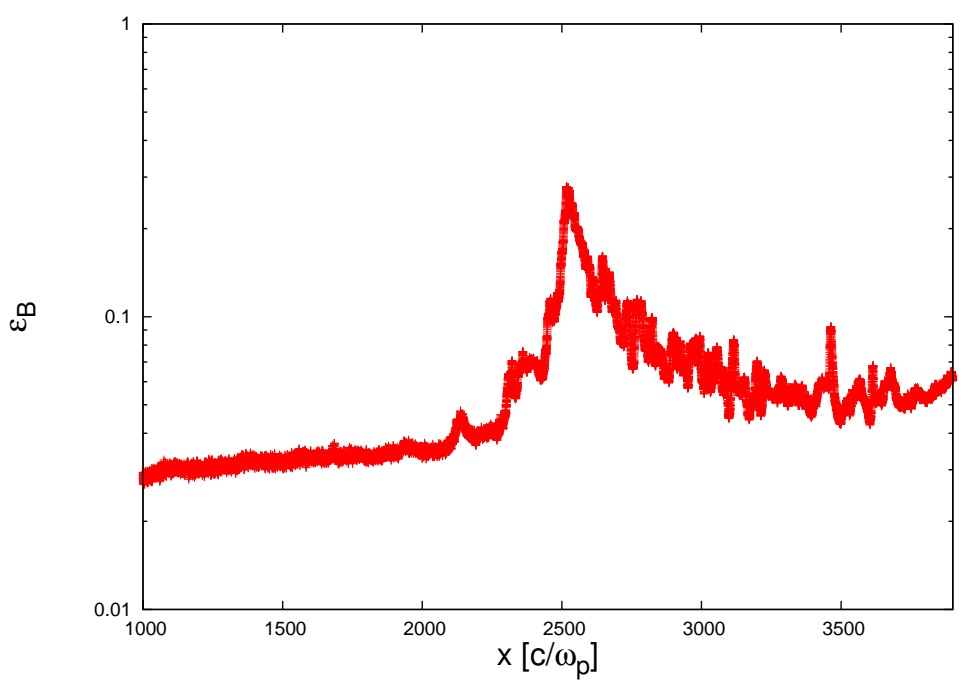

Figure 5: Spatial profile of the magnetic field energy density normalized by the mean upstream kinetic energy density, $\varepsilon_{\mathrm{B}}$ at $\omega_{\mathrm{p}} t=4000 . \varepsilon_{\mathrm{B}}$ is averaged over the y-direction. 


\section{Summary and Discussion}

We have performed the two-dimensional PIC simulation to investigate the magnetic-field generation in the downstream region of the collisionless shock propagating to the inhomogeneous plasma. The simulation show that the downstream region keeps the high temperature anisotropy for the inhomogeneous case, although the magnetic field simply decays in the downstream region. The observed temperature anisotropy is of the order of $10^{-2}$ which is large enough to generate the magnetic field expected from observations of GRB afterglows. However, according to the linear analysis, in order to observe the Weibel instability by the temperature anisotropy in this simulation, we have to perform the simulation for a longer time, $\omega_{\mathrm{p}} t \sim 10^{4}$. This will be addressed in future work.

\section{Acknowledgments}

We thank R. Yamazaki, S. Kisaka and J. Shimoda, for useful comments. The software used in this work was in part developed in pCANS at Chiba University. Numerical computations were carried out on Cray XC30 at Center for Computational Astrophysics, National Astronomical Observatory of Japan. This work is supported by Grant-in-Aid for JSPS Research Fellow from Japan Society for the Promotion of Science, No. 40420509. 


\section{References}

[1] A. D. Greenwiid, K. L. Cartwright, J. W. Luginsland, E. A. Baca, On the elimination of numerical Cerenkov radiation in PIC simulations, JCoPh. 201, 665-684, 2004.

[2] R. C. Davidson, D. A. Hammer, Haber, I., C. E. Wagner, Nonlinear Development of Electromagnetic Instabilities in Anisotropic Plasmas Phys. Fluild, 15, 317, 1972

[3] B. B. Godfrey, Numerical Cherenkov instabilities in electromagnetic particle codes, JCoPh., 15, 504, 1974.

[4] T. Kato, Nonrelativistic Collisionless Shock in Weakly Magnetized Electron-ion Plasmas: Two-Dimensional Particle-In-Cell Simulation of Perpendicular Shock, ApJ, 721, 828-842, 2010.

[5] P. Kumar and R. B. Duran, On the Generation of High-Energy Photons Detected by the Fermi Satellite from Gamma-Ray Bursts, MNRAS, 400, L75-L79, 2009.

[6] Y. Matsumoto, T. Amano, T. N. Kato, and M. Hoshino, Stochastic Electron Acceleration During Spontaneous Turbulent Reconnection in A Strong Shock Wave, Science, 347, 974-978, 2015.

[7] M. Medvedev and A. Loeb, Generation of Magnetic Fields in the Relativistic Shock of Gamma-ray Burst Sources, ApJ, 526, 697-706, 1999.

[8] pCANS, http://www.astro.phys.s.chiba-u.ac.jp/pcans/

[9] S. Tomita, Y. Ohira Weibel instability driven by spatially anisotropic density structures , ApJ, 825, 103-109, 2016

[10] Santana, R., Duran, R. B., Kumar, P., Magnetic fields in relativistic collisionless shocks, ApJ, 785, 29-47, 2014

[11] A. Spitkovsky, Particle Acceleration in Relativistic Collisionless Shocks: Fermi Process at Last?, ApJ, 682, L5-L9, 2008.

[12] J. -L. Vay, C. G. R. Geddes, C.-M. Estelle, D.P. Grote, Numerical methods for instability mitigation in the modeling of laser wakefield accelerators in a Lorentz-boosted frame, JCoPh. 230, 5908, 2011

[13] E. Waxman, Gamma-Ray-Burst Afterglow: Supporting the Cosmological Fireball Model, Constraining Parameters, and Making Predictions, ApJ, 485, L5-L8, 1997.

[14] E. S. Weibel, Spontaneously Growing Transverse Waves in a Plasma Due to an Anisotropic Velocity Distribution, PRL, 2, 83-84, 1959..... 